\title{
Using Stirling's Interpolation to Find Gauss and Mean Curvature for the Surface
}

\author{
R. B. M. Amer ${ }^{1}$, M. A. Abd El- Mageed ${ }^{2}$ \\ ${ }^{1,2}$ (Department of Mathematics and Phy. Engineering, Faculty of Engineering/Zagazig University, Egypt)
}

Abstract: In this paper, we can use Stirling's interpolation to compute the mean and gauss curvatures for any surface $z=f(x, y)$ at any point $\left(x_{0}, y_{0}\right)$.

Keywords: Differential geometry, Gauss curvature, Mathematica program, Mean curvature, Stirling's interpolation.

Surface characteristics can be divided into two types:

\section{Introduction}

1- Local characteristics are associated with points on the surface, and can be discovered by examining the local neighborhoods of points.

It is including:

- continuity

- mean curvature

- gaussian curvature

- singularities

- critical points: minima, maxima, and saddle points

2- Global characteristics are associated with the surface as a whole and cannot be determined strictly by looking at local neighborhoods

It is including:

- embeddedness

- orientability

- symmetry, including periodicity

- genus

- ends

- total curvature

- skeletal graphs

Definition 1.1. The gaussian curvature [1] of a surface at a point is the product of the principal curvatures $K=$ $\mathrm{k}_{1} \mathrm{k}_{2}$ at that point. The tangent plane of any point with positive gaussian curvature touches the surface at a single point, whereas the tangent plane of any point with negative gaussian curvature cuts the surface.

Definition 1.2.The mean curvature [1] half the sum of the principal curvatures $H=\left(k_{1}+k_{2}\right) / 2$, and any point with zero mean curvature has negative or zero gaussian curvature. Surfaces with zero mean curvature everywhere are minimal surfaces.

The gaussian and mean curvature play a very important role in the theory of surfaces and these are defined at each point on the surface.

Definition 1.3. The real-valued function of two variables $z=f(x, y)$ is a surface in $\mathfrak{R}^{3}$ [2].

\section{The Formulas of Gauss and Mean curvatures}

Let $\mathrm{k}_{1}, \mathrm{k}_{2}\left(k_{1} \geq k_{2}\right)$ denote the two principle curvature, the Gaussian curvature $\mathrm{K}$ and the mean curvature $\mathrm{H}$ are defined as $\mathrm{K}=\mathrm{k}_{1} \mathrm{k}_{2}$ and $\mathrm{H}=\frac{\mathbf{1}}{\mathbf{2}}\left(\mathbf{k}_{\mathbf{1}}+\mathbf{k}_{\mathbf{2}}\right)$. Formulas for computing $\mathrm{K}$ and $\mathrm{H}$ [3] are 


$$
\begin{aligned}
& \text { Mean curvature }=\mathrm{H}=\frac{\left(1+\mathrm{f}_{x}^{2}\right) \mathrm{f}_{\mathrm{yy}}+\left(1+\mathrm{f}_{y}^{2}\right) \mathrm{f}_{\mathrm{xx}}-2 \mathrm{f}_{\mathrm{x}} \mathrm{f}_{\mathrm{y}} \mathrm{f}_{\mathrm{xy}}}{2\left(1+\mathrm{f}_{\mathrm{x}}^{2}+\mathrm{f}_{\mathrm{y}}^{2}\right)^{\frac{3}{2}}}, \\
& \text { Gauss curvature }=\mathrm{K}=\frac{\mathrm{f}_{\mathrm{xx}}+\mathrm{f}_{\mathrm{yy}}-\mathrm{f}_{x y}^{2}}{\left(1+\mathrm{f}_{\mathrm{x}}^{2}+\mathrm{f}_{\mathrm{y}}^{2}\right)^{2}} .
\end{aligned}
$$

Therefore the principle curvature $\mathrm{k}_{1}, \mathrm{k}_{2}$ are

$$
\mathbf{k}_{1}=\mathbf{H}+\sqrt{\mathbf{H}^{2}-\mathbf{K}}, \mathbf{k}_{2}=\mathbf{H}-\sqrt{\mathbf{H}^{2}-\mathbf{K}} .
$$

We shall denote partial derivatives of functions by

$$
f_{x}=\frac{\partial f}{\partial x}, f_{x x}=\frac{\partial^{2} f}{\partial x^{2}}, f_{y}=\frac{\partial f}{\partial y}, f_{y y}=\frac{\partial^{2} f}{\partial y^{2}}, f_{x y}=\frac{\partial^{2} f}{\partial x \partial y} .
$$

\section{Using Mathematica Program}

From Mathematica program we can compute:

1.1. Expand $(\mathbf{a}+\mathbf{b})^{\mathbf{n}}$ from $\mathrm{n}=1,2,3,4,5,6,7,8,9,10$.

This is the program to evaluate the expand

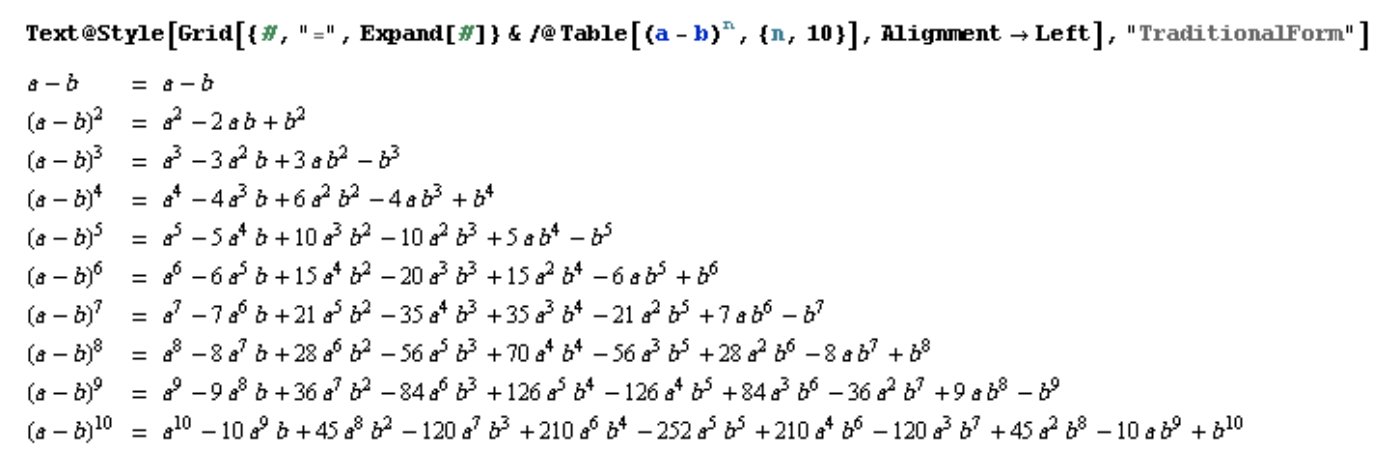

1.2. We use Do to write a procedural program for calculating the factorial of any integer by using a command fac to calculate the factorial of any positive integer $n$ and SetDelayed $(:=)$ for we do not want to evaluate the right side until fac is called.

$$
\begin{aligned}
& \text { fac }\left[n_{-}\right]:=(x=1 ; \operatorname{Do}[x=k * x,\{k, n\}] ; x) \\
& \text { TabView [Table [ToString }[n] " ! " \rightarrow \operatorname{fac}[n],\{n, 10\}], 10]
\end{aligned}
$$

\begin{tabular}{|l|l|l|l|l|l|l|l|l|l|l|}
\hline 11 & 12 & 13 & 14 & 15 & 16 & 17 & 18 & 19 & 110 \\
\hline 3628800 & & & & & & \\
\hline
\end{tabular}

Or use this easy command:

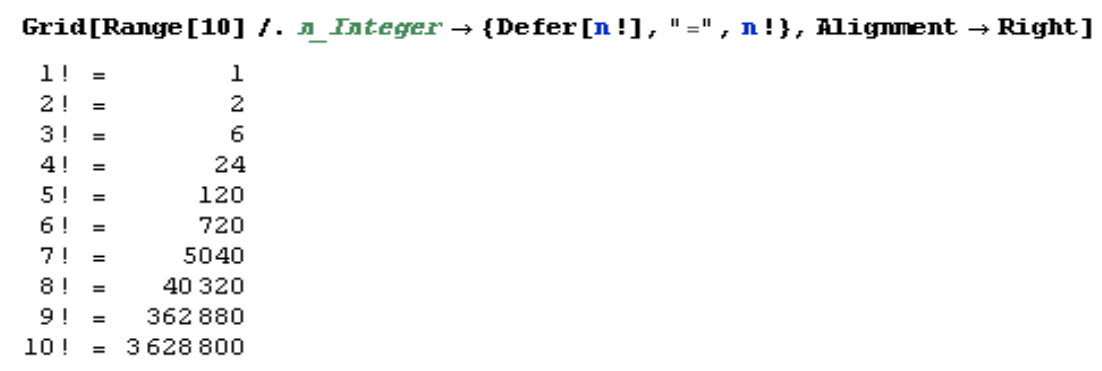

\section{Stirling's Central-Difference Interpolation}

Definition 4.1. Stirling's Central-Difference Interpolation formula is based on a diagonal difference table rather than a horizontal difference table. 
Without going in to the distinctions between the two types of difference tables, we can write down an interpolation formula including only second differences that is similar to Newton's interpolation formulas

Lemma 4.2. [4] Stirling's interpolation formula takes the form: [http://eom.springer.de/s/s087840.htm]

$\mathrm{f}_{\mathrm{p}}(\mathrm{x})=\mathrm{f}_{\mathrm{p}}\left(\mathrm{x}_{0}+\mathrm{ph}\right)=\mathrm{f}_{0}+\mathrm{pf}_{0}^{1}+\frac{\mathrm{p}^{2}}{2 !} \mathrm{f}_{0}^{2}+\ldots \ldots \ldots+\frac{\mathrm{p}\left(\mathrm{p}^{2}-1\right) \ldots .\left[\mathrm{p}^{2}-(\mathrm{n}-1)^{2}\right)}{(2 \mathrm{n}-1) !} \mathrm{f}_{0}^{2 \mathrm{n}-1}+$

$$
\frac{\mathrm{p}\left(\mathrm{p}^{2}-1\right) \ldots \cdot\left[\mathrm{p}^{2}-(\mathrm{n}-1)^{2}\right)}{2 \mathrm{n} !} \mathrm{f}_{0}^{2 \mathrm{n}} \text {. }
$$

Where $\quad \mathrm{p}=\left(\mathrm{x}-\mathrm{x}_{\mathrm{n}}\right) / \mathrm{h}=\left(\mathrm{x}-\mathrm{x}_{\mathrm{n}}\right) /\left(\mathrm{x}_{\mathrm{n}+1}-\mathrm{x}_{\mathrm{n}}\right)$

Since $\quad f_{p}(x)=f\left(x_{0}+p h\right)=f\left(x_{0}+p \Delta x\right)$.

Note that

$$
f\left(x_{0}\right)=f_{0}, f\left(x_{1}\right)=f_{1}, \ldots, f\left(x_{n}\right)=f_{n} .
$$

Remark 4.3. For small p, Stirling's interpolation formula is more exact than other interpolation formulas.

Definition 4.4. [4] The formula for any central difference $\delta$ can be

$\mathrm{f}_{\mathrm{n}+1 / 2}^{2 \mathrm{~m}+1}=\mathrm{f}_{\mathrm{n}+1}^{2 \mathrm{~m}}-\mathrm{f}_{\mathrm{n}}^{2 \mathrm{~m}}$,

$\mathrm{f}_{\mathrm{n}}^{2 \mathrm{~m}}=\mathrm{f}_{\mathrm{n}+1 / 2}^{2 \mathrm{~m}-1}-\mathrm{f}_{\mathrm{n}-1 / 2}^{2 \mathrm{~m}-1} . \quad(\mathrm{m}=0,1,2, \ldots ., \mathrm{n}=\ldots \ldots,-1,0,1, \ldots \ldots$.

We can write these equations as follows:

$\delta^{2 \mathrm{~m}} \mathrm{f}_{\mathrm{n}}=\delta^{2 \mathrm{~m}-1} \mathrm{f}_{\mathrm{n}+1 / 2}-\delta^{2 \mathrm{~m}-1} \mathrm{f}_{\mathrm{n}-1 / 2}$,

$\delta^{2 \mathrm{~m}+1} \mathrm{f}_{\mathrm{n}+1 / 2}=\delta^{2 \mathrm{~m}} \mathrm{f}_{\mathrm{n}+1}-\delta^{2 \mathrm{~m}} \mathrm{f}_{\mathrm{n}}$,

$\delta^{2 \mathrm{~m}+1} \mathrm{f}_{\mathrm{n}-1 / 2}=\delta^{2 \mathrm{~m}} \mathrm{f}_{\mathrm{n}}-\delta^{2 \mathrm{~m}_{\mathrm{n}-1}}$.

So $\mathrm{f}_{0}^{1}=\delta \mathrm{f}_{0}, \mathrm{f}_{0}^{2}=\delta^{2} \mathrm{f}_{0}, \mathrm{f}_{0}^{3}=\delta^{3} \mathrm{f}_{,} \mathrm{f}_{\mathrm{n}}^{\mathrm{m}}=\delta^{\mathrm{m}} \mathrm{f}_{\mathrm{n}}$ and so on.

Then

$$
\begin{aligned}
& \delta^{2} \mathrm{f}_{0}=\delta \mathrm{f}_{1 / 2}-\delta \mathrm{f}_{-1 / 2}=\mathrm{f}_{1}-\mathrm{f}_{0}-\mathrm{f}_{0}+\mathrm{f}_{-1}=\mathrm{f}_{1}-2 \mathrm{f}_{0}+\mathrm{f}_{-1} . \\
& \delta^{4} \mathrm{f}_{0}=\delta^{3} \mathrm{f}_{1 / 2}-\delta^{3} \mathrm{f}_{-1 / 2}=\delta^{2} \mathrm{f}_{1}-\delta^{2} \mathrm{f}_{0}-\delta^{2} \mathrm{f}_{0}+\delta^{2} \mathrm{f}_{-1}=\mathrm{f}_{2}-2 \mathrm{f}_{1}+\mathrm{f}_{0}-2 \mathrm{f}_{1}+4 \mathrm{f}_{0}-2 \mathrm{f}_{-1}+ \\
& +\mathrm{f}_{0}-2 \mathrm{f}_{-1}+\mathrm{f}_{-2} \text {. } \\
& \therefore \delta^{4} \mathrm{f}_{0}=\mathrm{f}_{2}-4 \mathrm{f}_{1}+6 \mathrm{f}_{0}-4 \mathrm{f}_{-1}+\mathrm{f}_{-2} \text {. } \\
& \delta^{6} \mathrm{f}_{0}=\delta^{5} \mathrm{f}_{1 / 2}-\delta^{5} \mathrm{f}_{-1 / 2}=\delta^{4} \mathrm{f}_{1}-\delta^{4} \mathrm{f}_{0}-\delta^{4} \mathrm{f}_{0}+\delta^{4} \mathrm{f}_{-1}=\mathrm{f}_{3}-4 \mathrm{f}_{2}+6 \mathrm{f}_{1}-4 \mathrm{f}_{0}+\mathrm{f}_{-1}-2 \mathrm{f}_{2}+8 \mathrm{f}_{1}- \\
& -12 \mathrm{f}_{0}+8 \mathrm{f}_{-1}-2 \mathrm{f}_{-2}+\mathrm{f}_{1}-4 \mathrm{f}_{0}+6 \mathrm{f}_{-1}-4 \mathrm{f}_{-2}+\mathrm{f}_{-3} \text {. } \\
& \therefore \delta^{6} \mathrm{f}_{0}=\mathrm{f}_{3}-6 \mathrm{f}_{2}+15 \mathrm{f}_{1}-20 \mathrm{f}_{0}+15 \mathrm{f}_{-1}+-6 \mathrm{f}_{-2}+\mathrm{f}_{-3} \text {. } \\
& \delta^{8} \mathrm{f}_{0}=\delta^{7} \mathrm{f}_{1 / 2}-\delta^{7} \mathrm{f}_{-1 / 2}=\delta^{6} \mathrm{f}_{1}-\delta^{6} \mathrm{f}_{0}-\delta^{6} \mathrm{f}_{0}+\delta^{6} \mathrm{f}_{-1}=\mathrm{f}_{4}-6 \mathrm{f}_{3}+15 \mathrm{f}_{2}-20 \mathrm{f}_{1}+15 \mathrm{f}_{0}-6 \mathrm{f}_{-1}+\mathrm{f}_{-2}- \\
& -2 \mathrm{f}_{3}+12 \mathrm{f}_{2}-30 \mathrm{f}_{1}+40 \mathrm{f}_{0}-30 \mathrm{f}_{-1}+12 \mathrm{f}_{-2}-2 \mathrm{f}_{-3}+ \\
& +\mathrm{f}_{2}-6 \mathrm{f}_{1}+15 \mathrm{f}_{0}-20 \mathrm{f}_{-1}+15 \mathrm{f}_{-2}-6 \mathrm{f}_{-3}+\mathrm{f}_{-4} \text {. } \\
& \therefore \delta^{8} \mathrm{f}_{0}=\mathrm{f}_{4}-8 \mathrm{f}_{3}+28 \mathrm{f}_{2}-56 \mathrm{f}_{1}+70 \mathrm{f}_{0}-56 \mathrm{f}_{-1}+28 \mathrm{f}_{-2}-8 \mathrm{f}_{-3}+\mathrm{f}_{-4} \text {. } \\
& \delta^{10} \mathrm{f}_{0}=\delta^{9} \mathrm{f}_{1 / 2}-\delta^{9} \mathrm{f}_{-1 / 2}=\delta^{8} \mathrm{f}_{1}-\delta^{8} \mathrm{f}_{0}-\delta^{8} \mathrm{f}_{0}+\delta^{8} \mathrm{f}_{-1}=\mathrm{f}_{5}-8 \mathrm{f}_{4}+28 \mathrm{f}_{3}-56 \mathrm{f}_{2}+70 \mathrm{f}_{1}-56 \mathrm{f}_{0}+28 \mathrm{f}_{-1}-8 \mathrm{f}_{-2}+\mathrm{f}_{-3}- \\
& -2 \mathrm{f}_{4}+16 \mathrm{f}_{3}-56 \mathrm{f}_{2}+112 \mathrm{f}_{1}-140 \mathrm{f}_{0}+112 \mathrm{f}_{-1}-56 \mathrm{f}_{-2}+16 \mathrm{f}_{-3}-2 \mathrm{f}_{-4}+ \\
& +\mathrm{f}_{3}-8 \mathrm{f}_{2}+28 \mathrm{f}_{1}-56 \mathrm{f}_{0}+70 \mathrm{f}_{-1}-56 \mathrm{f}_{-2}+28 \mathrm{f}_{-3}-8 \mathrm{f}_{-4}+\mathrm{f}_{-5} \text {. }
\end{aligned}
$$

$\therefore \delta^{10} \mathrm{f}_{0}=\mathrm{f}_{5}-10 \mathrm{f}_{4}+45 \mathrm{f}_{3}-120 \mathrm{f}_{2}+210 \mathrm{f}_{1}-252 \mathrm{f}_{0}+210 \mathrm{f}_{-1}-120 \mathrm{f}_{-2}+45 \mathrm{f}_{-3}-10 \mathrm{f}_{-4}+\mathrm{f}_{-5}$.

From [5]

$$
\mathrm{f}_{\mathrm{n}}^{2 \mathrm{k}-1}=\mu\left[\mathrm{f}_{\mathrm{n}+1 / 2}^{2 \mathrm{k}-1}+\mathrm{f}_{\mathrm{n}-1 / 2}^{2 \mathrm{k}-1}\right]=\frac{1}{2}\left[\mathrm{f}_{1 / 2}^{2 \mathrm{k}-1}+\mathrm{f}_{-1 / 2}^{2 \mathrm{k}-1}\right], \mathrm{k}=1,2,3, \ldots \ldots, \mathrm{n}=\ldots .,-1,0,1, \ldots \ldots
$$




$$
\mathrm{f}_{\mathrm{n}+1 / 2}^{2 k-1}=\mathrm{f}_{\mathrm{n}+1}^{2 k}-\mathrm{f}_{\mathrm{n}}^{2 k}
$$

Where $\mu[6]$ is averaging or mean operator defined by $\mu \mathrm{y}_{\mathrm{n}}=\frac{1}{2}\left[\mathrm{y}_{\mathrm{n}+\frac{1}{2}}+\mathrm{y}_{\mathrm{n}-\frac{1}{2}}\right]$

(17)

We can write these equations as follows:

$$
\begin{aligned}
& \delta^{2 k-1} f_{n}=\mu\left[\delta^{2 k-1} f_{n+1 / 2}-\delta^{2 k-1} f_{n-1 / 2}\right]=\frac{1}{2}\left[\delta^{2 k-1} f_{n+1 / 2}-\delta^{2 k-1} f_{n-1 / 2}\right], \\
& \delta^{2 k-1} f_{n+1 / 2}=\delta^{2 k-2} f_{n+1}-\delta^{2 k-2} f_{n}, \\
& \delta^{2 k-1} f_{n-1 / 2}=\delta^{2 k-2} f_{n}-\delta^{2 k-2} f_{n-1} .
\end{aligned}
$$

Then

$\delta \mathrm{f}_{0}=\frac{1}{2}\left[\mathrm{f}_{1 / 2}+\mathrm{f}_{-1 / 2}\right]=\frac{1}{2}\left[\mathrm{f}_{1}-\mathrm{f}_{0}+\mathrm{f}_{0}-\mathrm{f}_{-1}\right]=\frac{1}{2}\left[\mathrm{f}_{1}-\mathrm{f}_{-1}\right]$.

$\delta^{3} f_{0}=\frac{1}{2}\left[\delta^{3} f_{1 / 2}+\delta^{3} f_{-1 / 2}\right]$,

$\because \delta^{3} \mathrm{f}_{1 / 2}=\delta^{2} \mathrm{f}_{1}-\delta^{2} \mathrm{f}_{0}=\mathrm{f}_{2}-2 \mathrm{f}_{1}+\mathrm{f}_{0}-\mathrm{f}_{1}+2 \mathrm{f}_{0}-\mathrm{f}_{-1}=\mathrm{f}_{2}-3 \mathrm{f}_{1}+3 \mathrm{f}_{0}-\mathrm{f}_{-1}$,

$\because \delta^{3} \mathrm{f}_{-1 / 2}=\delta^{2} \mathrm{f}_{0}-\delta^{2} \mathrm{f}_{-1}=\mathrm{f}_{1}-2 \mathrm{f}_{0}+\mathrm{f}_{-1}-\mathrm{f}_{0}+2 \mathrm{f}_{-1}-\mathrm{f}_{-2}=\mathrm{f}_{1}-3 \mathrm{f}_{0}+3 \mathrm{f}_{-1}-\mathrm{f}_{-2}$,

$\therefore \delta^{3} \mathrm{f}_{0}=\frac{1}{2}\left[\mathrm{f}_{2}-2 \mathrm{f}_{1}+2 \mathrm{f}_{-1}-\mathrm{f}_{-2}\right]$.

$\delta^{5} \mathrm{f}_{0}=\frac{1}{2}\left[\delta^{5} \mathrm{f}_{1 / 2}+\delta^{5} \mathrm{f}_{-1 / 2}\right]$,

$\because \delta^{5} \mathrm{f}_{1 / 2}=\delta^{4} \mathrm{f}_{1}-\delta^{4} \mathrm{f}_{0} \quad=\mathrm{f}_{3}-4 \mathrm{f}_{2}+6 \mathrm{f}_{1}-4 \mathrm{f}_{0}+\mathrm{f}_{-1}-\mathrm{f}_{2}+4 \mathrm{f}_{1}-6 \mathrm{f}_{0}+4 \mathrm{f}_{-1}-\mathrm{f}_{-2}$

$=\mathrm{f}_{3}-5 \mathrm{f}_{2}+10 \mathrm{f}_{1}-10 \mathrm{f}_{0}+5 \mathrm{f}_{-1}-\mathrm{f}_{-2}$,

$\because \delta^{5} \mathrm{f}_{-1 / 2}=\delta^{4} \mathrm{f}_{0}-\delta^{4} \mathrm{f}_{-1}=\mathrm{f}_{2}-4 \mathrm{f}_{1}+6 \mathrm{f}_{0}-4 \mathrm{f}_{-1}+\mathrm{f}_{-2}-\mathrm{f}_{1}+4 \mathrm{f}_{0}-6 \mathrm{f}_{-1}+4 \mathrm{f}_{-2}-\mathrm{f}_{-3}$

$$
=\mathrm{f}_{2}-5 \mathrm{f}_{1}+10 \mathrm{f}_{0}-10 \mathrm{f}_{-1}+5 \mathrm{f}_{-2}-\mathrm{f}_{-3} \text {, }
$$

$\therefore \delta^{5} \mathrm{f}_{0}=\frac{1}{2}\left[\mathrm{f}_{3}-4 \mathrm{f}_{2}+5 \mathrm{f}_{1}-5 \mathrm{f}_{-1}+4 \mathrm{f}_{-2}-\mathrm{f}_{-3}\right]$.

$\delta^{7} f_{0}=\frac{1}{2}\left[\delta^{7} f_{1 / 2}+\delta^{7} f_{-1 / 2}\right]$,

$\because \delta^{7} \mathrm{f}_{1 / 2}=\delta^{6} \mathrm{f}_{1}-\delta^{6} \mathrm{f}_{0}=\mathrm{f}_{4}-6 \mathrm{f}_{3}+15 \mathrm{f}_{2}-20 \mathrm{f}_{1}+15 \mathrm{f}_{0}-6 \mathrm{f}_{-1}+\mathrm{f}_{-2}-$

$-f_{3}+6 f_{2}-15 f_{1}+20 f_{0}-15 f_{-1}+6 f_{-2}-f_{-3}$

$=f_{4}-7 f_{3}+21 f_{2}-35 f_{1}+35 f_{0}-21 f_{-1}+7 f_{-2}-f_{-3}$,

$\because \delta^{7} \mathrm{f}_{-1 / 2}=\delta^{6} \mathrm{f}_{0}-\delta^{6} \mathrm{f}_{-1}=\mathrm{f}_{3}-6 \mathrm{f}_{2}+15 \mathrm{f}_{1}-20 \mathrm{f}_{0}+15 \mathrm{f}_{-1}-6 \mathrm{f}_{-2}+\mathrm{f}_{-3}-$

$-\mathrm{f}_{2}+6 \mathrm{f}_{1}-15 \mathrm{f}_{0}+20 \mathrm{f}_{-1}-15 \mathrm{f}_{-2}+6 \mathrm{f}_{-3}-\mathrm{f}_{-4}$

$=f_{3}-7 f_{2}+21 f_{1}-35 f_{0}+35 f_{-1}-21 f_{-2}+7 f_{-3}-f_{-4}$,

$\therefore \delta^{7} f_{0}=\frac{1}{2}\left[f_{4}-6 f_{3}+14 f_{2}-14 f_{1}+14 f_{-1}-14 f_{-2}+6 f_{-3}-f_{-4}\right]$.

$\delta^{9} \mathrm{f}_{0}=\frac{1}{2}\left[\delta^{9} \mathrm{f}_{1 / 2}+\delta^{9} \mathrm{f}_{-1 / 2}\right]$,

$\because \delta^{9} \mathrm{f}_{1 / 2}=\delta^{8} \mathrm{f}_{1}-\delta^{8} \mathrm{f}_{0} \quad=\mathrm{f}_{5}-8 \mathrm{f}_{4}+28 \mathrm{f}_{3}-56 \mathrm{f}_{2}+70 \mathrm{f}_{1}-56 \mathrm{f}_{0}+28 \mathrm{f}_{-1}-8 \mathrm{f}_{-2}+\mathrm{f}_{-3}-$

$-\mathrm{f}_{4}+8 \mathrm{f}_{3}-28 \mathrm{f}_{2}+56 \mathrm{f}_{1}-70 \mathrm{f}_{0}+56 \mathrm{f}_{-1}-28 \mathrm{f}_{-2}+8 \mathrm{f}_{-3}-\mathrm{f}_{-4}$

$=\mathrm{f}_{5}-9 \mathrm{f}_{4}+36 \mathrm{f}_{3}-84 \mathrm{f}_{2}+126 \mathrm{f}_{1}-126 \mathrm{f}_{0}+84 \mathrm{f}_{-1}-36 \mathrm{f}_{-2}+9 \mathrm{f}_{-3}-\mathrm{f}_{-4}$,

$\because \delta^{9} \mathrm{f}_{-1 / 2}=\delta^{8} \mathrm{f}_{0}-\delta^{8} \mathrm{f}_{-1}=\mathrm{f}_{4}-8 \mathrm{f}_{3}+28 \mathrm{f}_{2}-56 \mathrm{f}_{1}+70 \mathrm{f}_{0}-56 \mathrm{f}_{-1}+28 \mathrm{f}_{-2}-8 \mathrm{f}_{-3}+\mathrm{f}_{-4}-$

$-\mathrm{f}_{3}+8 \mathrm{f}_{2}-28 \mathrm{f}_{1}+56 \mathrm{f}_{0}-70 \mathrm{f}_{-1}+56 \mathrm{f}_{-2}-28 \mathrm{f}_{-3}+8 \mathrm{f}_{-4}-\mathrm{f}_{-5}$

$=\mathrm{f}_{4}-9 \mathrm{f}_{3}+36 \mathrm{f}_{2}-84 \mathrm{f}_{1}+126 \mathrm{f}_{0}-126 \mathrm{f}_{-1}+84 \mathrm{f}_{-2}-36 \mathrm{f}_{-3}+9 \mathrm{f}_{-4}-\mathrm{f}_{-5}$,

$\therefore \delta^{9} \mathrm{f}_{0}=\frac{1}{2}\left[\mathrm{f}_{5}-8 \mathrm{f}_{4}+27 \mathrm{f}_{3}-48 \mathrm{f}_{2}+42 \mathrm{f}_{1}-42 \mathrm{f}_{-1}+48 \mathrm{f}_{-2}-27 \mathrm{f}_{-3}+8 \mathrm{f}_{-4}-\mathrm{f}_{-5}\right]$. 
Thus given values of a function $f(x)$ at a finite number of discrete points $n=10$, and letting $p=\left[\left(x_{-}-x_{i}\right) /\left(x_{i+1}-\right.\right.$ $\left.\mathrm{x}_{\mathrm{i}}\right)$ ], Stirling's central-difference interpolation formula can be expressed using functional values as

$$
\begin{aligned}
\mathrm{f}_{\mathrm{p}}= & \mathrm{f}_{0}+\mathrm{p} \delta f_{0}+\frac{\mathrm{p}^{2}}{2 !} \delta^{2} \mathrm{f}_{0}+\frac{\mathrm{p}\left(\mathrm{p}^{2}-1\right)}{3 !} \delta^{3} \mathrm{f}_{0}+\frac{\mathrm{p}^{2}\left(\mathrm{p}^{2}-1\right)}{4 !} \delta^{4} \mathrm{f}_{0}+\frac{\mathrm{p}\left(\mathrm{p}^{2}-1\right)\left(\mathrm{p}^{2}-4\right)}{5 !} \delta^{5} \mathrm{f}_{0}+ \\
& +\frac{\mathrm{p}^{2}\left(\mathrm{p}^{2}-1\right)\left(\mathrm{p}^{2}-4\right)}{6 !} \delta^{6} \mathrm{f}_{0}+\frac{\mathrm{p}\left(\mathrm{p}^{2}-1\right)\left(\mathrm{p}^{2}-4\right)\left(\mathrm{p}^{2}-9\right)}{7 !} \delta^{7} \mathrm{f}_{0}+\frac{\mathrm{p}^{2}\left(\mathrm{p}^{2}-1\right)\left(\mathrm{p}^{2}-4\right)\left(\mathrm{p}^{2}-9\right)}{8 !} \delta^{8} \mathrm{f}_{0}+ \\
& +\frac{\mathrm{p}\left(\mathrm{p}^{2}-1\right)\left(\mathrm{p}^{2}-4\right)\left(\mathrm{p}^{2}-9\right)\left(\mathrm{p}^{2}-16\right)}{9 !} \delta^{9} \mathrm{f}_{0}+\frac{\mathrm{p}^{2}\left(\mathrm{p}^{2}-1\right)\left(\mathrm{p}^{2}-4\right)\left(\mathrm{p}^{2}-9\right)\left(\mathrm{p}^{2}-16\right)}{10 !} \delta^{10} \mathrm{f}_{0} .
\end{aligned}
$$

From above equations, we can write this equation as

$$
\begin{aligned}
& \mathrm{f}_{\mathrm{p}}= \mathrm{f}_{0}+\frac{\mathrm{p}}{2}\left[\mathrm{f}_{1}-\mathrm{f}_{-1}\right]+\frac{\mathrm{p}^{2}}{2}\left[\mathrm{f}_{1}-2 \mathrm{f}_{0}+\mathrm{f}_{-1}\right]+\frac{\mathrm{p}\left(\mathrm{p}^{2}-1\right)}{12}\left[\mathrm{f}_{2}-2 \mathrm{f}_{1}+2 \mathrm{f}_{-1}-\mathrm{f}_{-2}\right]+ \\
&+\frac{\mathrm{p}^{2}\left(\mathrm{p}^{2}-1\right)}{24}\left[\mathrm{f}_{2}-4 \mathrm{f}_{1}+6 \mathrm{f}_{0}-4 \mathrm{f}_{-1}+\mathrm{f}_{-2}\right]+ \\
&+\frac{\mathrm{p}\left(\mathrm{p}^{2}-1\right)\left(\mathrm{p}^{2}-4\right)}{240}\left[\mathrm{f}_{3}-4 \mathrm{f}_{2}+5 \mathrm{f}_{1}-5 \mathrm{f}_{-1}+4 \mathrm{f}_{-2}-\mathrm{f}_{-3}\right]+ \\
&+\frac{\mathrm{p}^{2}\left(\mathrm{p}^{2}-1\right)\left(\mathrm{p}^{2}-4\right)}{720}\left[\mathrm{f}_{3}-6 \mathrm{f}_{2}+15 \mathrm{f}_{1}-20 \mathrm{f}_{\mathrm{o}}+15 \mathrm{f}_{-1}+-6 \mathrm{f}_{-2}+\mathrm{f}_{-3}\right] \\
&+\frac{\mathrm{p}\left(\mathrm{p}^{2}-1\right)\left(\mathrm{p}^{2}-4\right)\left(\mathrm{p}^{2}-9\right)}{10080}\left[\mathrm{f}_{4}-6 \mathrm{f}_{3}+14 \mathrm{f}_{2}-14 \mathrm{f}_{1}+14 \mathrm{f}_{-1}-14 \mathrm{f}_{-2}+6 \mathrm{f}_{-3}-\mathrm{f}_{-4}\right]+ \\
&+\frac{\mathrm{p}^{2}\left(\mathrm{p}^{2}-1\right)\left(\mathrm{p}^{2}-4\right)\left(\mathrm{p}^{2}-9\right)}{40320}\left[\mathrm{f}_{4}-8 \mathrm{f}_{3}+28 \mathrm{f}_{2}-56 \mathrm{f}_{1}+70 \mathrm{f}_{0}-56 \mathrm{f}_{-1}+28 \mathrm{f}_{-2}-8 \mathrm{f}_{-3}+\mathrm{f}_{-4}\right]+ \\
&+\frac{p\left(\mathrm{p}^{2}-1\right)\left(\mathrm{p}^{2}-4\right)\left(\mathrm{p}^{2}-9\right)\left(\mathrm{p}^{2}-16\right)}{725760}\left[\mathrm{f}_{5}-8 \mathrm{f}_{4}+27 \mathrm{f}_{3}-48 \mathrm{f}_{2}+42 \mathrm{f}_{1}-42 \mathrm{f}_{-1}+48 \mathrm{f}_{-2}-27 \mathrm{f}_{-3}+\right] \\
&+\frac{\mathrm{p}^{2}\left(\mathrm{p}^{2}-1\right)\left(\mathrm{p}^{2}-4\right)\left(\mathrm{p}^{2}-9\right)\left(\mathrm{p}^{2}-16\right)}{3628800}\left[\mathrm{f}_{5}-10 \mathrm{f}_{-5}+45 \mathrm{f}_{3}-120 \mathrm{f}_{2}+210 \mathrm{f}_{1}-252 \mathrm{f}_{0}+210 \mathrm{f}_{-1}-\right] . \\
&-120 \mathrm{f}_{-2}+45 \mathrm{f}_{-3}-10 \mathrm{f}_{-4}+\mathrm{f}_{-5}
\end{aligned}
$$

Differentiate this equation with respect to $\mathrm{p}$ and put $\mathrm{p}=0$, then

$$
\begin{aligned}
& \left.\quad \frac{\mathrm{df}\left(\mathrm{x}_{\mathrm{p}}, \mathrm{y}_{0}\right)}{\mathrm{dp}}\right|_{\left(\mathrm{x}_{0}, \mathrm{y}_{0}\right)}=\left.\mathrm{f}_{\mathrm{p}}^{\prime}\right|_{\left(\mathrm{x}_{0}, \mathrm{y}_{0}\right) \mathrm{p}=0} \\
& \begin{aligned}
&\left.\therefore \mathrm{f}_{\mathrm{p}}^{\prime}\right|_{\left(\mathrm{x}_{0}, \mathrm{y}_{0}\right), \mathrm{p}=0}=\frac{1}{2}\left[\mathrm{f}_{1}-\mathrm{f}_{-1}\right]-\frac{1}{12}\left[\mathrm{f}_{2}-2 \mathrm{f}_{1}+2 \mathrm{f}_{-1}-\mathrm{f}_{-2}\right]+ \\
& \quad+\frac{1}{60}\left[\mathrm{f}_{3}-4 \mathrm{f}_{2}+5 \mathrm{f}_{1}-5 \mathrm{f}_{-1}+4 \mathrm{f}_{-2}-\mathrm{f}_{-3}\right]- \\
& \quad-\frac{1}{280}\left[\mathrm{f}_{4}-6 \mathrm{f}_{3}+14 \mathrm{f}_{2}-14 \mathrm{f}_{1}+14 \mathrm{f}_{-1}-14 \mathrm{f}_{-2}+6 \mathrm{f}_{-3}-\mathrm{f}_{-4}\right]+ \\
& \quad+\frac{1}{1260}\left[\mathrm{f}_{5}-8 \mathrm{f}_{4}+27 \mathrm{f}_{3}-48 \mathrm{f}_{2}+42 \mathrm{f}_{1}-42 \mathrm{f}_{-1}+48 \mathrm{f}_{-2}-27 \mathrm{f}_{-3}++8 \mathrm{f}_{-4}-\mathrm{f}_{-5}\right] \\
& \text { Then } \quad \\
&\left.\mathrm{f}_{\mathrm{p}}^{\prime}\right|_{\mathrm{p}=0}= 7.9365 \times 10^{-4}\left(\mathrm{f}_{5}-\mathrm{f}_{-5}\right)-9.92063 \times 10^{-3}\left(\mathrm{f}_{4}-\mathrm{f}_{-4}\right)+0.0553571\left(\mathrm{f}_{3}-\mathrm{f}_{-3}\right)- \\
&-0.238095\left(\mathrm{f}_{2}-\mathrm{f}_{-2}\right)+0.75\left(\mathrm{f}_{1}-\mathrm{f}_{-1}\right)
\end{aligned}
\end{aligned}
$$

Since $\mathrm{x}=\mathrm{x}_{0}+\mathrm{ph}=\mathrm{x}_{\mathrm{p}}$.

Then $\frac{\mathrm{dx}}{\mathrm{dp}}=\mathrm{h}, \frac{\mathrm{dp}}{\mathrm{dx}}=\frac{1}{\mathrm{~h}}$

We get $\quad \frac{d f\left(x, y_{0}\right)}{d x}=f_{x}=\frac{d f\left(x, y_{0}\right)}{d p} \times \frac{d p}{d x}=f_{p}^{\prime} X \frac{d p}{d x}$

Let the interval length $\mathrm{h}=0.001$

Then we can get $\mathrm{f}_{\mathrm{x}}$ at a point $\left(\mathrm{x}_{0}, \mathrm{y}_{0}\right)$ for the surface $\mathrm{z}=\mathrm{f}(\mathrm{x}, \mathrm{y})$

$$
\mathrm{f}_{\mathrm{x}}=100\left[\begin{array}{c}
7.9365 \times 10^{-4}\left(\mathrm{f}_{5}-\mathrm{f}_{-5}\right)-9.92063 \times 10^{-3}\left(\mathrm{f}_{4}-\mathrm{f}_{-4}\right)+0.0553571\left(\mathrm{f}_{3}-\mathrm{f}_{-3}\right)- \\
-0.238095\left(\mathrm{f}_{2}-\mathrm{f}_{-2}\right)+0.75\left(\mathrm{f}_{1}-\mathrm{f}_{-1}\right)
\end{array}\right] .
$$


Differentiate $\mathrm{f}_{\mathrm{p}}$ twice with respect to $\mathrm{p}$ and put $\mathrm{p}=0$, then

$$
\begin{aligned}
& \left.\frac{\mathrm{d}^{2} \mathrm{f}\left(\mathrm{x}_{\mathrm{p}}, \mathrm{y}_{0}\right)}{\mathrm{dp}^{2}}\right|_{\left(\mathrm{x}_{0}, \mathrm{y}_{0}\right)}=\left.\mathrm{f}_{\mathrm{p}}^{\prime \prime}\right|_{\left(\mathrm{x}_{0}, \mathrm{y}_{0}\right), \mathrm{p}=0} \\
& \left.\therefore \mathrm{f}_{\mathrm{p}}^{\prime \prime}\right|_{\left(\mathrm{x}_{0}, \mathrm{y}_{0}\right), \mathrm{p}=0}=\left[\mathrm{f}_{1}-2 \mathrm{f}_{\mathrm{o}}+\mathrm{f}_{-1}\right]-\frac{1}{12}\left[\mathrm{f}_{2}-4 \mathrm{f}_{1}+6 \mathrm{f}_{0}-4 \mathrm{f}_{-1}+\mathrm{f}_{-2}\right]+ \\
& +\frac{1}{90}\left[\mathrm{f}_{3}-6 \mathrm{f}_{2}+15 \mathrm{f}_{1}-20 \mathrm{f}_{0}+15 \mathrm{f}_{-1}+-6 \mathrm{f}_{-2}+\mathrm{f}_{-3}\right]- \\
& -\frac{1}{560}\left[\mathrm{f}_{4}-8 \mathrm{f}_{3}+28 \mathrm{f}_{2}-56 \mathrm{f}_{1}+70 \mathrm{f}_{0}-56 \mathrm{f}_{-1}+28 \mathrm{f}_{-2}-8 \mathrm{f}_{-3}+\mathrm{f}_{-4}\right]+ \\
& +\frac{1}{3150}\left[\mathrm{f}_{5}-10 \mathrm{f}_{4}+45 \mathrm{f}_{3}-120 \mathrm{f}_{2}+210 \mathrm{f}_{1}-252 \mathrm{f}_{0}+210 \mathrm{f}_{-1}-120 \mathrm{f}_{-2}+45 \mathrm{f}_{-3}-10 \mathrm{f}_{-4}+\mathrm{f}_{-5}\right] \\
& \text { Then } \\
& \left.\mathrm{f}_{\mathrm{p}}^{\prime \prime}\right|_{\mathrm{p}=\mathrm{O}}=3.17 \times 10^{-4}\left(\mathrm{f}_{5}-\mathrm{f}_{-5}\right)-4.96 \times 10^{-3}\left(\mathrm{f}_{4}-\mathrm{f}_{-4}\right)+0.039683\left(\mathrm{f}_{3}-\mathrm{f}_{-3}\right)- \\
& -0.238095\left(f_{2}-f_{-2}\right)+1.666667\left(f_{1}-f_{-1}\right)-2.927222 f_{0} \text {. }
\end{aligned}
$$

Then we can get $\mathrm{f}_{\mathrm{xx}}$ at a point $\left(\mathrm{x}_{0}, \mathrm{y}_{0}\right)$ for the surface $\mathrm{z}=\mathrm{f}(\mathrm{x}, \mathrm{y})$

$$
\mathrm{f}_{\mathrm{xx}}=(100)^{2}\left[\begin{array}{r}
3.17 \times 10^{-4}\left(\mathrm{f}_{5}-\mathrm{f}_{-5}\right)-4.96 \times 10^{-3}\left(\mathrm{f}_{4}-\mathrm{f}_{-4}\right)+0.039683\left(\mathrm{f}_{3}-\mathrm{f}_{-3}\right)- \\
-0.238095\left(\mathrm{f}_{2}-\mathrm{f}_{-2}\right)+1.666667\left(\mathrm{f}_{1}-\mathrm{f}_{-1}\right)-2.927222 \mathrm{f}_{0}
\end{array}\right] .
$$

We can also find $\mathrm{f}_{\mathrm{y}}$ and $\mathrm{f}_{\mathrm{y}}$ at a point $\left(\mathrm{x}_{0}, \mathrm{y}_{0}\right)$ for the surface $\mathrm{z}=\mathrm{f}(\mathrm{x}, \mathrm{y})$ by using the same method

Since $\left.\frac{\mathrm{df}\left(\mathrm{x}_{0}, \mathrm{y}_{\mathrm{p}}\right)}{\mathrm{dp}}\right|_{\left(\mathrm{x}_{0}, \mathrm{y}_{0}\right)}=\left.\tilde{\mathrm{f}}_{\mathrm{p}}^{\prime}\right|_{\left(\mathrm{x}_{0}, \mathrm{y}_{0}\right), \mathrm{p}=0},\left.\frac{\mathrm{d}^{2} \mathrm{f}\left(\mathrm{x}_{0}, \mathrm{y}_{\mathrm{p}}\right)}{\mathrm{dp}^{2}}\right|_{\left(\mathrm{x}_{0}, \mathrm{y}_{0}\right)}=\left.\tilde{\mathrm{f}}_{\mathrm{p}}^{\prime \prime}\right|_{\left(\mathrm{x}_{0}, \mathrm{y}_{0}\right), \mathrm{p}=0}$

Then we get

$$
\mathrm{f}_{\mathrm{y}}=100\left[\begin{array}{c}
7.9365 \times 10^{-4}\left(\tilde{\mathrm{f}}_{5}-\widetilde{\mathrm{f}}_{-5}\right)-9.92063 \times 10^{-3}\left(\tilde{\mathrm{f}}_{4}-\widetilde{\mathrm{f}}_{-4}\right)+0.0553571\left(\tilde{\mathrm{f}}_{3}-\widetilde{\mathrm{f}}_{-3}\right)- \\
-0.238095\left(\tilde{\mathrm{f}}_{2}-\widetilde{\mathrm{f}}_{-2}\right)+0.75\left(\tilde{\mathrm{f}}_{1}-\widetilde{\mathrm{f}}_{-1}\right)
\end{array}\right] \text {. }
$$

and

$$
\mathrm{f}_{\mathrm{yy}}=(100)^{2}\left[\begin{array}{r}
3.17 \times 10^{-4}\left(\tilde{\mathrm{f}}_{5}-\tilde{\mathrm{f}}_{-5}\right)-4.96 \times 10^{-3}\left(\tilde{\mathrm{f}}_{4}-\tilde{\mathrm{f}}_{-4}\right)+0.039683\left(\tilde{\mathrm{f}}_{3}-\tilde{\mathrm{f}}_{-3}\right)- \\
-0.238095\left(\tilde{\mathrm{f}}_{2}-\tilde{\mathrm{f}}_{-2}\right)+1.666667\left(\tilde{\mathrm{f}}_{1}-\tilde{\mathrm{f}}_{-1}\right)-2.927222 \tilde{\mathrm{f}}_{0}
\end{array}\right] .
$$

To calculate $\mathrm{f}_{\mathrm{xy}}$ at a point $\left(\mathrm{x}_{0}, \mathrm{y}_{0}\right)$ for the surface $\mathrm{z}=\mathrm{f}(\mathrm{x}, \mathrm{y})$ by Stirling's central-difference interpolation formula which expressed using functional values as

$$
\begin{aligned}
\mathrm{f}_{\mathrm{p}}=\mathrm{f}\left(\mathrm{x}_{0}+\mathrm{ph}, \mathrm{y}\right) & =\mathrm{f}\left(\mathrm{x}_{0}, \mathrm{y}\right)+\mathrm{p} \delta f\left(x_{0}, y\right)+\frac{\mathrm{p}^{2}}{2 !} \delta^{2} \mathrm{f}\left(\mathrm{x}_{0}, \mathrm{y}\right)+\frac{\mathrm{p}\left(\mathrm{p}^{2}-1\right)}{3 !} \delta^{3} \mathrm{f}\left(\mathrm{x}_{0}, \mathrm{y}\right)+\frac{\mathrm{p}^{2}\left(\mathrm{p}^{2}-1\right)}{4 !} \delta^{4} \mathrm{f}\left(\mathrm{x}_{0}, \mathrm{y}\right)+ \\
& +\frac{\mathrm{p}\left(\mathrm{p}^{2}-1\right)\left(\mathrm{p}^{2}-4\right)}{5 !} \delta^{5} \mathrm{f}\left(\mathrm{x}_{0}, \mathrm{y}\right)+\frac{\mathrm{p}^{2}\left(\mathrm{p}^{2}-1\right)\left(\mathrm{p}^{2}-4\right)}{6 !} \delta^{6} \mathrm{f}\left(\mathrm{x}_{0}, \mathrm{y}\right)+ \\
& +\frac{\mathrm{p}\left(\mathrm{p}^{2}-1\right)\left(\mathrm{p}^{2}-4\right)\left(\mathrm{p}^{2}-9\right)}{7 !} \delta^{7} \mathrm{f}\left(\mathrm{x}_{0}, \mathrm{y}\right)+\frac{\mathrm{p}^{2}\left(\mathrm{p}^{2}-1\right)\left(\mathrm{p}^{2}-4\right)\left(\mathrm{p}^{2}-9\right)}{8 !} \delta^{8} \mathrm{f}\left(\mathrm{x}_{0}, \mathrm{y}\right)+ \\
& +\frac{\mathrm{p}\left(\mathrm{p}^{2}-1\right)\left(\mathrm{p}^{2}-4\right)\left(\mathrm{p}^{2}-9\right)\left(\mathrm{p}^{2}-16\right)}{9 !} \delta^{9} \mathrm{f}\left(\mathrm{x}_{0}, \mathrm{y}\right)+\frac{\mathrm{p}^{2}\left(\mathrm{p}^{2}-1\right)\left(\mathrm{p}^{2}-4\right)\left(\mathrm{p}^{2}-9\right)\left(\mathrm{p}^{2}-16\right)}{10 !} \delta^{10} \mathrm{f}\left(\mathrm{x}_{0}, \mathrm{y}\right) .
\end{aligned}
$$

Differentiate this equation with respect to $p$, then 


$$
\begin{aligned}
& \frac{\left.\mathrm{df}\left(\mathrm{x}_{\mathrm{p}}, \mathrm{y}\right)\right|_{\left(\mathrm{x}_{0}, \mathrm{y}\right)}=\left.\mathrm{f}_{\mathrm{p}}^{\prime}\right|_{\left(\mathrm{x}_{0}, \mathrm{y}\right)}}{\mathrm{dp}} \\
& \therefore \mathrm{f}_{\mathrm{p}}^{\prime}=\mathrm{f}_{p}^{\prime}\left(\mathrm{x}_{0}+\mathrm{ph}, \mathrm{y}\right)=\delta f\left(x_{0}, y\right)+p \delta^{2} \mathrm{f}\left(\mathrm{x}_{0}, \mathrm{y}\right)+\frac{3 \mathrm{p}^{2}-1}{6} \delta^{3} \mathrm{f}\left(\mathrm{x}_{0}, \mathrm{y}\right)+\frac{4 \mathrm{p}^{3}-2 p}{24} \delta^{4} \mathrm{f}\left(\mathrm{x}_{0}, \mathrm{y}\right)+ \\
& +\frac{5 p^{4}-15 p^{2}+4}{120} \delta^{5} \mathrm{f}\left(\mathrm{x}_{0}, \mathrm{y}\right)+\frac{6 p^{5}-20 p^{3}+8 p}{720} \delta^{6} \mathrm{f}\left(\mathrm{x}_{0}, \mathrm{y}\right)+ \\
& +\frac{7 p^{6}-70 p^{4}+147 p^{2}-36}{5040} \delta^{7} \mathrm{f}\left(\mathrm{x}_{0}, \mathrm{y}\right)+\frac{8 p^{7}-84 p^{5}+196 p^{3}-72 p}{40320} \delta^{8} \mathrm{f}\left(\mathrm{x}_{0}, \mathrm{y}\right)+ \\
& +\frac{9 p^{8}-210 p^{6}+1365 p^{4}-2460 p^{2}+576}{362880} \delta^{9} \mathrm{f}\left(\mathrm{x}_{0}, \mathrm{y}\right)+ \\
& +\frac{10 p^{9}-240 p^{7}+1638 p^{5}-3280 p^{3}+1152 p}{3628800} \delta^{10} \mathrm{f}\left(\mathrm{x}_{0}, \mathrm{y}\right) .
\end{aligned}
$$

Let $y=y_{0}+q h, f\left(x_{0}, y_{0}\right)=f_{0}$

Then

$$
\begin{aligned}
& \mathrm{f}_{p}^{\prime}\left(\mathrm{x}_{0}+p h, y_{0}+q h\right)=\delta\left[\begin{array}{l}
\tilde{\mathrm{f}}_{0}+q \delta \tilde{\mathrm{f}}_{0}+\frac{q^{2}}{2} \delta^{2} \widetilde{\mathrm{f}}_{0}+\frac{q^{3}-q}{6} \delta^{3} \widetilde{\mathrm{f}}_{0^{+}} \\
+\frac{q^{4}-q^{2}}{24} \delta^{4} \widetilde{\mathrm{f}}_{0^{+}}+\frac{q^{5}-5 q^{3}+4 q}{120} \delta^{5} \widetilde{\mathrm{f}}_{0^{+}} \\
+\frac{q^{6}-5 q^{4}+4 q^{2}}{720} \delta^{6} \widetilde{\mathrm{f}}_{0}+ \\
+\frac{q^{7}-14 q^{5}+49 q^{3}-36 q}{5040} \delta^{7} \widetilde{\mathrm{f}}_{0^{+}} \\
+\frac{q^{8}-14 q^{6}+49 q^{4}-36 q^{2}}{40320} \delta^{8} \widetilde{\mathrm{f}}_{0}+ \\
+\frac{q^{9}-30 q^{7}+273 q^{5}-820 q^{3}+576 q}{362880} \delta^{9} \widetilde{\mathrm{f}}_{0}+ \\
+\frac{q^{10}-30 q^{8}+273 q^{6}-820 q^{4}+576 q^{2}}{3628800} \delta^{10} \widetilde{\mathrm{f}}_{0}
\end{array}\right]+ \\
& +p \delta^{2}\left[\begin{array}{l}
\tilde{\mathrm{f}}_{0}+q \delta \tilde{\mathrm{f}}_{0}+\frac{q^{2}}{2} \delta^{2} \widetilde{\mathrm{f}}_{0}+\frac{q^{3}-q}{6} \delta^{3} \widetilde{\mathrm{f}}_{0}+ \\
+\frac{q^{4}-q^{2}}{24} \delta^{4} \widetilde{\mathrm{f}}_{0}+\frac{q^{5}-5 q^{3}+4 q}{120} \delta^{5} \widetilde{\mathrm{f}}_{0}+ \\
+\frac{q^{6}-5 q^{4}+4 q^{2}}{720} \delta^{6} \widetilde{\mathrm{f}}_{0}+ \\
+\frac{q^{7}-14 q^{5}+49 q^{3}-36 q}{5040} \delta^{7} \widetilde{\mathrm{f}}_{0^{+}} \\
+\frac{q^{8}-14 q^{6}+49 q^{4}-36 q^{2}}{40320} \delta^{8} \widetilde{\mathrm{f}}_{0^{+}} \\
+\frac{q^{9}-30 q^{7}+273 q^{5}-820 q^{3}+576 q}{362880} \delta^{9} \widetilde{\mathrm{f}}_{0}+ \\
+\frac{q^{10}-30 q^{8}+273 q^{6}-820 q^{4}+576 q^{2}}{3628800} \delta^{10} \widetilde{\mathrm{f}}_{0}
\end{array}\right]+
\end{aligned}
$$

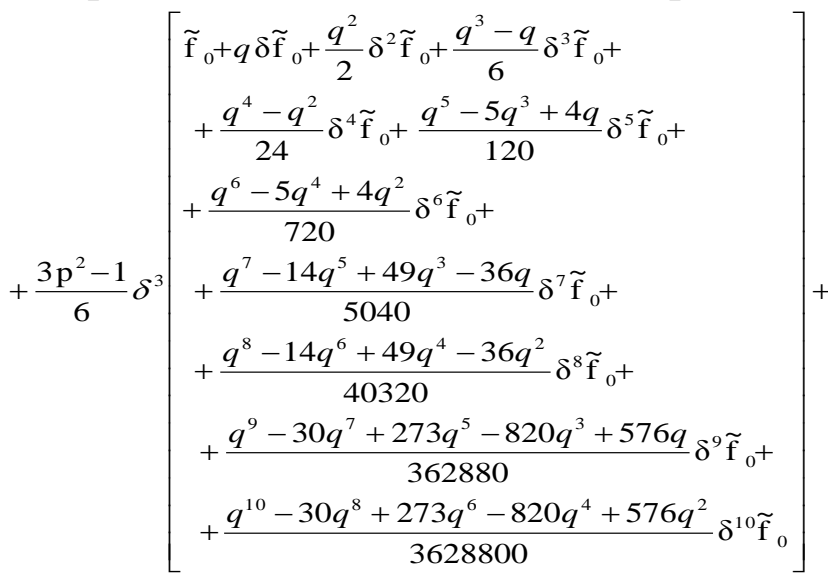




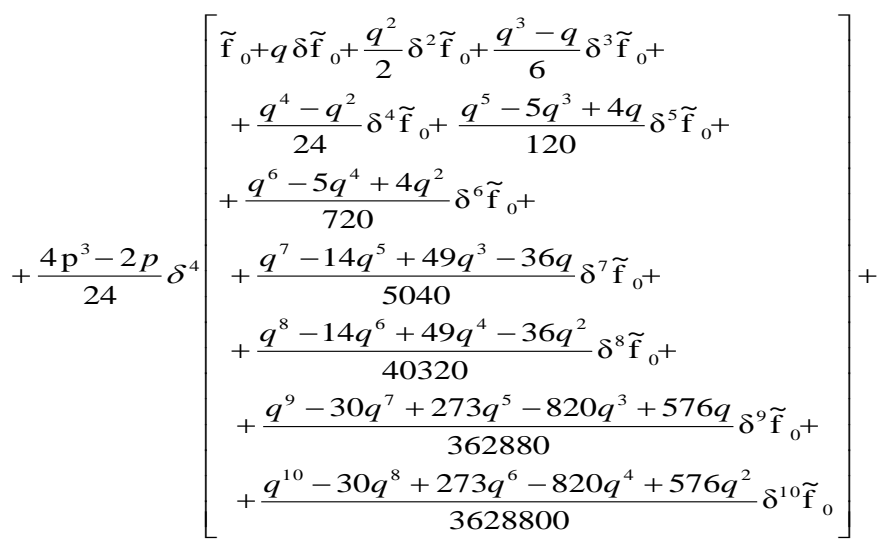

$+\frac{5 p^{4}-15 p^{2}+4}{120} \delta^{5}\left[\begin{array}{l}+\frac{q^{6}-5 q^{4}+4 q^{2}}{720} \delta^{6} \widetilde{\mathbf{f}}_{0^{+}}+q \delta \widetilde{\mathbf{f}}_{0}+\frac{q^{2}}{2} \delta^{2} \widetilde{\mathbf{f}}_{0}+\frac{q^{3}-q}{6} \delta^{3} \widetilde{\mathbf{f}}_{0^{+}} \\ +\frac{q^{4}-q^{2}}{24} \delta^{4} \widetilde{\mathbf{f}}_{0^{2}}+\frac{q^{5}-5 q^{3}+4 q}{5040} \delta^{5} \widetilde{\mathbf{f}}_{0^{+}} \\ +\frac{q^{8}-14 q^{6}+49 q^{4}-36 q^{2}}{40320} \delta^{8} \widetilde{\mathbf{f}}_{0^{+}} \\ +\frac{q^{9}-30 q^{7}+273 q^{5}-820 q^{3}+576 q}{362880} \delta^{9} \widetilde{\mathbf{f}}_{0^{+}} \\ +\frac{q^{10}-30 q^{8}+273 q^{6}-820 q^{4}+576 q^{2}}{3628800} \delta^{10} \widetilde{\mathbf{f}}_{0}\end{array}\right]+$

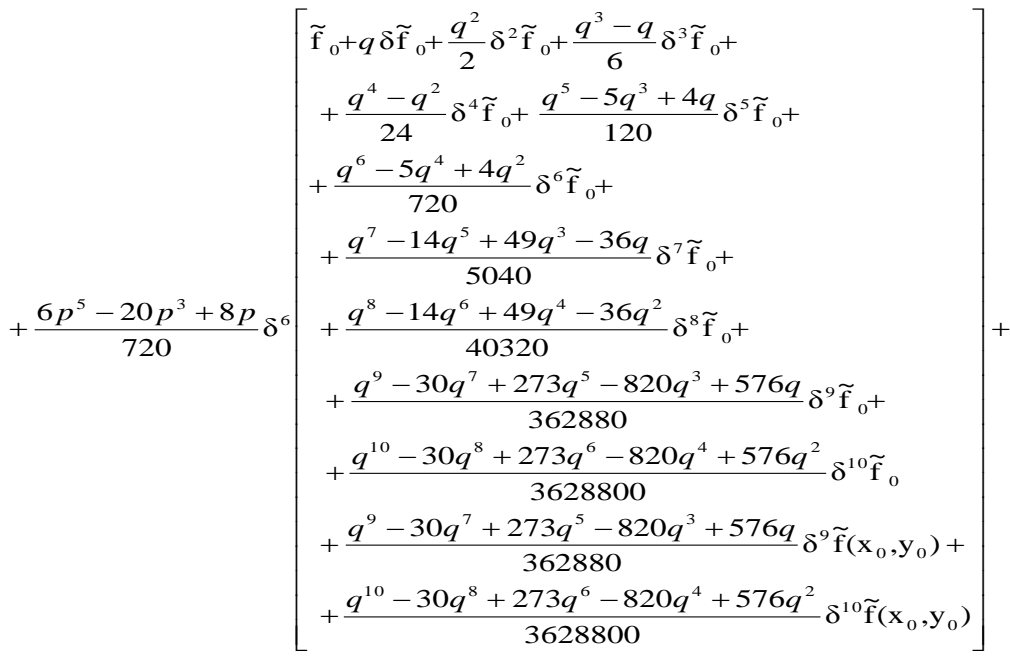

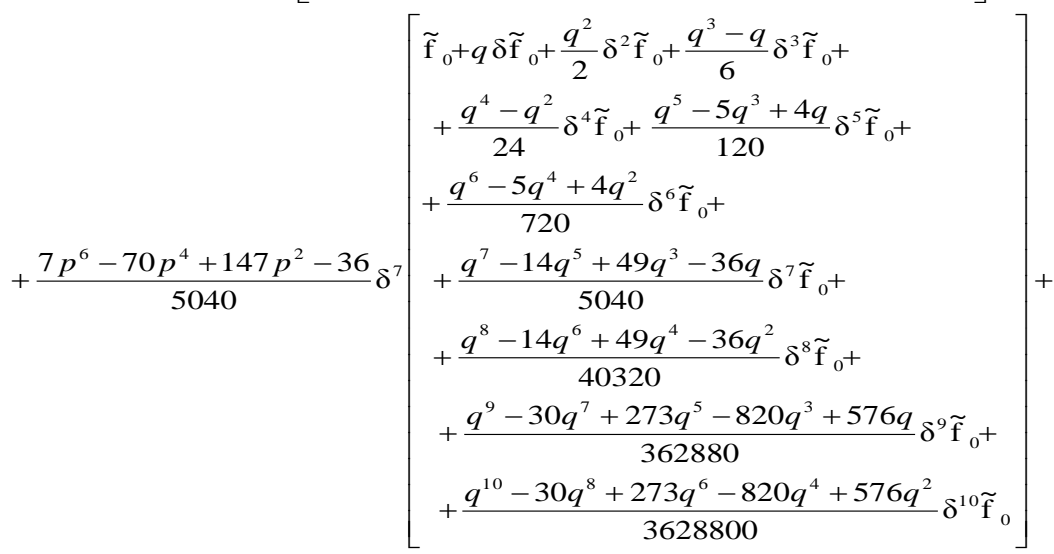




$$
\begin{aligned}
& +\frac{8 p^{7}-84 p^{5}+196 p^{3}-72 p}{40320} \delta^{8}\left[\begin{array}{l}
\widetilde{\mathrm{f}}_{0}+q \delta \widetilde{\mathbf{f}}_{0}+\frac{q^{2}}{2} \delta^{2} \widetilde{\mathbf{f}}_{0}+\frac{q^{3}-q}{6} \delta^{3} \widetilde{\mathrm{f}}_{0^{+}} \\
+\frac{q^{4}-q^{2}}{24} \delta^{4} \widetilde{\mathrm{f}}_{0^{+}}+\frac{q^{5}-5 q^{3}+4 q}{120} \delta^{5} \widetilde{\mathrm{f}}_{0^{+}} \\
+\frac{q^{6}-5 q^{4}+4 q^{2}}{720} \delta^{6} \widetilde{\mathrm{f}}_{0^{+}} \\
+\frac{q^{7}-14 q^{5}+49 q^{3}-36 q}{5040} \delta^{7} \widetilde{\mathrm{f}}_{0^{+}} \\
+\frac{q^{8}-14 q^{6}+49 q^{4}-36 q^{2}}{40320} \delta^{8} \widetilde{\mathbf{f}}_{0}+ \\
+\frac{q^{9}-30 q^{7}+273 q^{5}-820 q^{3}+576 q}{362880} \delta^{9} \widetilde{\mathrm{f}}_{0}+ \\
+\frac{q^{10}-30 q^{8}+273 q^{6}-820 q^{4}+576 q^{2}}{3628800} \delta^{10} \widetilde{\mathbf{f}}_{0}
\end{array}\right]+
\end{aligned}
$$

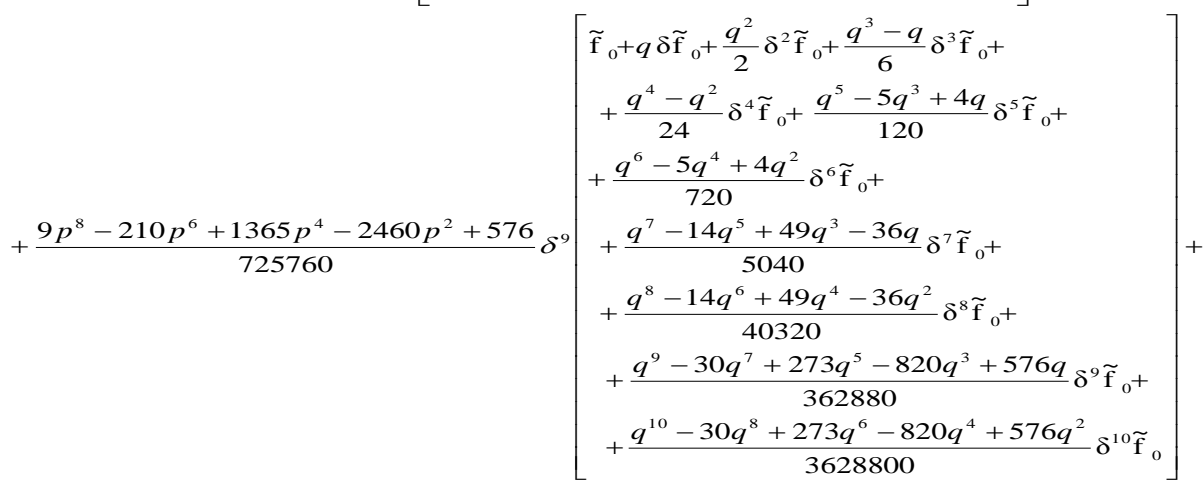

$$
\begin{aligned}
& +\frac{10 p^{9}-240 p^{7}+1638 p^{5}-3280 p^{3}+1152 p}{3628800} \delta^{10}\left[\begin{array}{l}
\widetilde{\mathrm{f}}_{0}+q \delta \widetilde{\mathrm{f}}_{0}+\frac{q^{2}}{2} \delta^{2} \widetilde{\mathbf{f}}_{0^{+}} \frac{q^{3}-q}{6} \delta^{3} \widetilde{\mathrm{f}}_{0^{+}} \\
+\frac{q^{4}-q^{2}}{24} \delta^{4} \widetilde{\mathrm{f}}_{0^{+}} \frac{q^{5}-5 q^{3}+4 q}{120} \delta^{5} \widetilde{\mathrm{f}}_{0^{+}} \\
+\frac{q^{6}-5 q^{4}+4 q^{2}}{720} \delta^{6} \widetilde{\mathrm{f}}_{0^{+}} \\
+\frac{q^{7}-14 q^{5}+49 q^{3}-36 q}{5040} \delta^{7} \widetilde{\mathrm{f}}_{0^{+}} \\
+\frac{q^{8}-14 q^{6}+49 q^{4}-36 q^{2}}{40320} \delta^{8} \widetilde{\mathrm{f}}_{0^{+}} \\
+\frac{q^{9}-30 q^{7}+273 q^{5}-820 q^{3}+576 q}{36280} \delta^{9} \widetilde{\mathrm{f}}_{0^{+}} \\
+\frac{q^{10}-30 q^{8}+273 q^{6}-820 q^{4}+576 q^{2}}{3628800} \delta^{10} \widetilde{\mathrm{f}}_{0}
\end{array}\right]
\end{aligned}
$$

Differentiate $f_{p}$ twice with respect to $p, q$ and put $p=0, q=0$ then

$$
\begin{aligned}
& \left.\mathrm{f}_{\mathrm{p}}^{\prime \prime}\right|_{\left(\mathrm{x}_{0}, \mathrm{y}_{0}\right), \mathrm{p}=0, q=0}=\delta\left[\delta \widetilde{f}_{0}-\frac{1}{6} \delta^{3} \widetilde{f}_{0}+\frac{1}{30} \delta^{5} \tilde{f}_{0}-\frac{1}{140} \delta^{7} \tilde{f}_{0}+\frac{1}{630} \delta^{9} \tilde{f}_{0}\right]- \\
& -\frac{1}{6} \delta^{3}\left[\delta \tilde{f}_{0}-\frac{1}{6} \delta^{3} \tilde{f}_{0}+\frac{1}{30} \delta^{5} \tilde{f}_{0}-\frac{1}{140} \delta^{7} \tilde{f}_{0}+\frac{1}{630} \delta^{9} \tilde{f}_{0}\right]+ \\
& +\frac{1}{30} \delta^{5}\left[\delta \tilde{f}_{0}-\frac{1}{6} \delta^{3} \widetilde{f}_{0}+\frac{1}{30} \delta^{5} \widetilde{f}_{0}-\frac{1}{140} \delta^{7} \tilde{f}_{0}+\frac{1}{630} \delta^{9} \widetilde{f}_{0}\right]- \\
& -\frac{1}{140} \delta^{7}\left[\delta \tilde{f}_{0}-\frac{1}{6} \delta^{3} \tilde{f}_{0}+\frac{1}{30} \delta^{5} \tilde{f}_{0}-\frac{1}{140} \delta^{7} \tilde{f}_{0}+\frac{1}{630} \delta^{9} \tilde{f}_{0}\right]+ \\
& +\frac{1}{630} \delta^{9}\left[\delta \tilde{f}_{0}-\frac{1}{6} \delta^{3} \tilde{f}_{0}+\frac{1}{30} \delta^{5} \tilde{f}_{0}-\frac{1}{140} \delta^{7} \tilde{f}_{0}+\frac{1}{630} \delta^{9} \tilde{f}_{0}\right]
\end{aligned}
$$

We get:

$$
\begin{aligned}
\left.\mathrm{f}_{\mathrm{p}}^{\prime \prime}\right|_{\left(\mathrm{x}_{0}, \mathrm{y}_{0}\right), \mathrm{p}=0, q=0}= & \delta^{2} \tilde{f}_{\mathrm{o}}+\delta^{4} \tilde{f}_{\mathrm{o}}\left(-\frac{1}{6}-\frac{1}{6}\right)+\delta^{6} \tilde{f}_{\mathrm{o}}\left(\frac{1}{30}+\frac{1}{36}+\frac{1}{30}\right)+ \\
& +\delta^{8} \tilde{f}_{\mathrm{o}}\left(-\frac{1}{140}-\frac{1}{180}-\frac{1}{140}-\frac{1}{180}\right)+ \\
& +\delta^{10} \tilde{f}_{\mathrm{o}}\left(\frac{1}{630}+\frac{1}{840}+\frac{1}{900}+\frac{1}{840}+\frac{1}{630}\right) .
\end{aligned}
$$

Then, 


$$
\begin{aligned}
\left.\mathrm{f}_{\mathrm{p}}^{\prime \prime}\right|_{\left(\mathrm{x}_{0}, y_{0}\right), \mathrm{p}=0, q=0}= & \tilde{f}_{1}-2 \tilde{f}_{0}+\tilde{f}_{-1}-0.33333\left[\tilde{f}_{2}-4 \tilde{f}_{1}+6 \tilde{f}_{0}-4 \tilde{f}_{-1}+\tilde{f}_{-2}\right]_{+} \\
& +0.09444\left[\tilde{f}_{3}-6 \tilde{f}_{2}+15 \tilde{f}_{1}-20 \tilde{f}_{0}+15 \tilde{f}_{-1}-6 \tilde{f}_{-2}+\tilde{f}_{-3}\right]_{-} \\
& -0.0254\left[\tilde{f}_{4}-8 \tilde{f}_{3}+28 \tilde{f}_{2}-56 \tilde{f}_{1}+70 \tilde{f}_{0}-56 \tilde{f}_{-1}+28 \tilde{f}_{-2}-8 \tilde{f}_{-3}+\tilde{f}_{-4}\right]+ \\
& +6.66667 \times 10^{-3}\left[\begin{array}{l}
\tilde{f}_{5}-10 \tilde{f}_{4}+45 \tilde{f}_{3}-120 \tilde{f}_{2}+210 \tilde{f}_{1}-252 \tilde{f}_{0}+210 \tilde{f}_{-1}- \\
-120 \tilde{f}_{-2}+45 \tilde{f}_{-3}-10 \tilde{f}_{-4}+\tilde{f}_{-5} .
\end{array}\right] .
\end{aligned}
$$

Hence,

$$
\begin{aligned}
\left.\mathrm{f}_{\mathrm{p}}^{\prime \prime}\right|_{\left(\mathrm{x}_{0}, y_{0}\right), \mathrm{p}=0, q=0}= & -7.83562 \widetilde{f}_{\mathrm{o}}+5.31302\left(\widetilde{f}_{1}+\widetilde{f}_{-1}\right)-1.69157\left(\widetilde{f}_{2}+\widetilde{f}_{-2}\right)+ \\
& +0.32779\left[\widetilde{f}_{3}+\widetilde{f}_{-3}\right]-0.0321\left[\widetilde{f}_{4}+\widetilde{f}_{-4}\right]+6.66667 \times 10^{-3}\left[\widetilde{f}_{5}+\widetilde{f}_{-5} .\right]
\end{aligned}
$$

Let $\mathrm{h}=0.001$

Then we can get $\mathrm{f}_{\mathrm{xy}}$ at a point $\left(\mathrm{x}_{0}, \mathrm{y}_{0}\right)$ for the surface $\mathrm{z}=\mathrm{f}(\mathrm{x}, \mathrm{y})$

$$
f_{x y}=(100)^{2}\left[\begin{array}{l}
-7.83562 \tilde{f}_{0}+5.31302\left(\tilde{f}_{1}+\tilde{f}_{-1}\right)-1.69157\left(\tilde{f}_{2}+\tilde{f}_{-2}\right)+ \\
\left.+0.32779\left[\tilde{f}_{3}+\tilde{f}_{-3}\right]-0.0321\left[\tilde{f}_{4}+\tilde{f}_{-4}\right]+6.66667 x 10^{-3}\left[\tilde{f}_{5}+\tilde{f}_{-5} \cdot\right] \cdot\right]
\end{array}\right]
$$

\section{Conclusions}

The most important central difference formula is stirling interpolation and it takes the mean of Gauss forward and backward formulae. Stirling 's formula gives the most accurate result for small $\mathrm{p}$ and interpolation near the middle of a table.

\section{References}

[1] K. Wolfgang, Differential geometry: curves - surfaces - manifolds ( American Mathematical Society, ISBN 0821839888, 2006).

[2] O. John, Differential geometry and its applications ( Rrentice - Hall, Inc., U.S.A, 1997).

[3] H. Adam, M. Ronald \& K. Harab, 2005, Surface curvature estimation for automatic colonic polyp detection, Proceedings of SPIE, Bellingham, WA, 2005, Vol. 5746.

[4] F. B. Hildebrand, Introduction to Numerical Analysis ( New York: Dover, 1974,Second edition).

[5] I.S. Berezin and N.P. Zhidkov, Computing methods ( Pergamon (Translated from Russian), 1973).

[6] S. S. Sastry, Introductory Methods of Numerical Analysis (4th. Ed prentice Hall of India, NewDelhi, 2005). 